\title{
Reseña bibliográfica de: “Kreimer, P. (2019). Science and Society in Latin America. Peripheral Modernities. New York: Routledge. 270 pp."
}

Palabras claves: Historia y Sociología de la Ciencia; Campos Científicos; Centros/periferias.

Keywords: History and Sociology of Science; Scientific Fields; Centers/Peripheries.

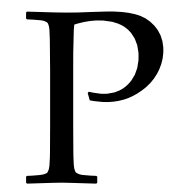

ablo Kreimer en Science and Society in Latin America reúne una serie de trabajos, que, si bien han circulado previamente en distintas publicaciones, al ser conectados entre sí nos proponen comprender la historia de la ciencia en los países latinoamericanos a partir de su desarrollo en el marco de "modernidades periféricas". En ese sentido, el autor nos invita a reconocer la especificidad de la experiencia de quienes han desarrollado investigación científica en el contexto de América Latina, pero adoptando "los valores, prácticas, creencias e ideas de 'ciencia'" propias de sus pares europeos y norteamericanos. Para Kreimer, estos científicos han vivido la aparente paradoja de ser modernos y periféricos al mismo tiempo y, por ese medio, constructores de una "ciencia periférica", con sus dinámicas, problemáticas y trayectorias singulares.

Para analizar las particularidades de la producción y el uso de los saberes científicos en América Latina y las modalidades en que sus practicantes se integraron en el campo científico internacional, el autor adopta como unidad de análisis las prácticas académicas llevadas a cabo por grupos de elite, fundamentalmente en biomedicina, física y biología, en países que institucionalizaron políticas científicas desde finales del siglo XIX. Más precisamente, focaliza su atención en la trayectoria de grupos argentinos y, en menor medida, analiza algunos casos brasileños y chilenos. Ese criterio de selección se fundamenta en que esos países, si bien tempranamente adhirieron a las concepciones modernas que sustentaron el despliegue de prácticas científicas, al estar insertos en contextos periféricos, tuvieron un desenvolvimiento distinto al modelo estándar de desarrollo científico.

La obra se inicia con un capítulo que funciona de estado de la cuestión respecto a la historiografía y la sociología del conocimiento científico y su utilidad para aprehender campos como los latinoamericanos. En contraposición a los distintos paradigmas vigentes, el autor propone pensar en centros y periferias en plural y adopta algunas claves interpretativas: la relevancia asignada al contexto en la explicación de la producción y el 
uso del conocimiento; el estudio de las relaciones internacionales y locales que entablan los actores pertenecientes a las comunidades científicas; la definición de los contenidos y alcances de la negociación entre los actores involucrados. El abordaje de esas claves analíticas se lleva a cabo metodológicamente mediante la adopción de una perspectiva microanalítica de las comunidades científicas, siguiendo mediante un exhaustivo trabajo de reconstrucción empírica la trayectoria de agentes e instituciones.

Los siguientes capítulos desarrollan los diferentes ejes destinados a dilucidar cómo se construyen los problemas públicos y científicos, cómo se institucionalizan los campos disciplinares y cómo se internacionalizan los saberes en el marco de las prácticas científicas periféricas.

El tercer capítulo está dedicado al primer eje relativo a cómo se construye un problema epistémico y un problema público a partir del análisis del conocimiento científico relativo a la enfermedad de Chagas. A lo largo de ese capítulo, Kreimer nos propone recorrer la manera en que alrededor de esta enfermedad, científicos, políticos, laboratorios y enfermos han tenido recursos y posibilidades diversas para influir en cómo el Chagas ha sido construido como un problema público, pero también para definir qué tipo de intervención era necesaria sobre la enfermedad. Para ahondar en esta cuestión, en el capítulo 4, presenta diversos esquemas teóricos que intentan explicar el rol de los científicos en la construcción de los problemas públicos y, retomando a Joseph Gusfield, destaca la importancia de analizar el lugar que adquiere la retórica científica en esa labor. Estas discusiones son profundizadas en el capítulo 6, dedicado al análisis de las controversias respecto al tratamiento de los enfermos crónicos de Chagas, temática a partir de la cual se indaga en la manera en que "los discursos y los mecanismos que validan la demostración científica son construidos" (p. 148) y negociados al interior de la comunidad científica.

El siguiente eje que recuperamos del libro es el que remite a las claves para interpretar el desarrollo de los campos científicos en la ciencia periférica. Inicialmente, en el análisis se introducen dimensiones contextuales relativas a la creación e institucionalización de una práctica científica, pero mostrando cómo estos factores políticos y económicos operan de manera diferenciada y no automática. De tal modo, no todas las disrupciones habrían funcionado de manera semejante, ni de forma uniforme sobre los diversos actores e instituciones.

Una cuestión medular en el desenvolvimiento de esos campos periféricos fueron las relaciones internacionales entre grupos de investigación a través de la transferencia de problemas y técnicas novedosas. Justamente a lo largo del libro se busca interpretar cuál ha sido el impacto de las relaciones entre centros y periferias en las actividades y productos de la ciencia periférica. Según el autor, las comunidades latinoamericanas han tendido a vincularse en los campos científicos a nivel internacional mediante una "integración subordinada". Este fenómeno se manifiesta en la manera en que las agendas de investigación han sido modeladas por las prioridades impuestas por los centros científicos internacionales en donde se localizan los mecanismos de validación más 
valorados. Esto ha derivado en la orientación de las comunidades científicas locales hacia la producción de conocimiento aplicable, pero no aplicado, lo que para Kreimer "es un verdadero indicador de la naturaleza estructuralmente periférica de la cultura científica local" (p. 79).

El concepto de "integración subordinada" es trabajado más extensamente en dos capítulos más. En el capítulo 7, se analiza el desenvolvimiento del fenómeno a instancias de los cambios que se producen con la internacionalización de la ciencia, producto de modificaciones en los grupos centrales y en las relaciones que entablan con las comunidades locales de científicos. En tiempos de mega redes de investigación, en proyectos en los que participan centenares $\mathrm{o}$, incluso, miles de investigadores en consorcios internacionales, los practicantes de la ciencia periférica tienen pocas posibilidades de incidir en la definición epistémica de los problemas de investigación, reduciendo su contribución a la instancia técnica de producción de datos. Por su parte, en el capítulo 8, a partir de la perspectiva de científicos localizados en campos periféricos, se indaga en las razones subyacentes al establecimiento de este tipo de vínculos, sus condicionamientos y limitaciones.

En definitiva, a través del análisis en profundidad de casos de grupos científicos latinoamericanos exitosos, el libro de Pablo Kreimer propone generalizaciones tendientes a identificar regularidades en su desenvolvimiento, periodizaciones en la generación de conocimiento científico y tipologías útiles en la comprensión de relaciones académicas norte/sur.

María José Ortiz Bergia Universidad Nacional de Córdoba - Consejo Nacional de Investigaciones Científicas y Técnicas ortizbergia.mj@gmail.com

Para citar este artículo:

Ortiz Bergia, María José (2020). Reseña bibliográfica: “Kreimer, P. (2019). Science and Society in Latin America. Peripheral Modernities. New York: Routledge. 270 pp." Anuario de la Escuela de Historia Virtual, 18, 281-283. 